Research Article

\title{
Cooperative Attitude Control of Multiple Rigid Bodies with Multiple Time-Varying Delays and Dynamically Changing Topologies
}

\author{
Ziyang Meng, ${ }^{1}$ Zheng You, $^{2}$ Guanhua Li, ${ }^{1}$ and Chunshi Fan ${ }^{1}$ \\ ${ }^{1}$ Department of Precision Instruments and Mechanology, Tsinghua University, Beijing 100084, China \\ 2 State Key Laboratory of Precision Measurement Technology and Instruments, Tsinghua University, \\ Beijing 100084, China \\ Correspondence should be addressed to Ziyang Meng, panday227@gmail.com
}

Received 2 January 2010; Revised 20 April 2010; Accepted 1 June 2010

Academic Editor: Tamas Kalmar-Nagy

Copyright (c) 2010 Ziyang Meng et al. This is an open access article distributed under the Creative Commons Attribution License, which permits unrestricted use, distribution, and reproduction in any medium, provided the original work is properly cited.

\begin{abstract}
Cooperative attitude regulation and tracking problems are discussed in the presence of multiple time-varying communication delays and dynamically changing topologies. In the case of cooperative attitude regulation, we propose conditions to guarantee the stability of the closed-loop system when there exist multiple time-varying communication delays. In the case of cooperative attitude tracking, the result of uniformly ultimate boundedness of the closed-loop system is obtained when there exist both multiple time-varying communication delays and dynamically changing topologies. Simulation results are presented to validate the effectiveness of these conclusions.
\end{abstract}

\section{Introduction}

Cooperative control of multiagent system has been followed with extensive interest in recent years. Compared to single-agent system, greater benefits such as greater efficiency, lower costs, and higher robustness can be realized by cooperation of multiagent system. The basic idea of cooperative control of multiagent system is that each agent in the group uses its local interactions such that the common objectives and tasks can be achieved [1]. One important application toward this direction is distributed cooperative attitude control for multiple rigid bodies. In particular, in the context of deep space interferometry, it is often necessary and significant to maintain relative attitude synchronization precisely during and after maneuvers among a formation of spacecraft $[2,3]$, where cooperative attitude control may serve as an effective tool.

As a decentralized control strategy, cooperative attitude control demonstrates many superior qualities compared with the traditionally centralized approaches. A good survey on 
cooperative attitude control can be found in [4]. In particular, a leader-following structure was used in $[2,5]$, where the follower spacecraft are assumed to have access to the information of the leader spacecraft. The authors in [6] proposed a behavioral strategy to realize attitude synchronization, where the behavior of individual spacecraft attitude tracking and that of formation keeping were considered together in an index function. As an alternative to the behavioral strategy, the virtual structure approach was proposed in [7], where the entire formation is treated as a single rigid body. The communication topology was highlighted in [8-10] and the attitude coordination problems were presented by using relative attitude and relative angular velocity information. Attitude containment control for multiple stationary leaders were considered in [11], where the linearly relative attitude was expressed by using Modified Rodriguez Parameters (MRPs) as attitude representation.

Although lots of benefits can be obtained from cooperative control of multiagent system, the performance of such networked system is often subject to communication failure and communication delays. Plenty results on the influence of delays and dynamically changing topologies have been obtained for multiagent system described by simplified models of motion, such as single-integrator dynamics and double-integrator dynamics. In particular, the authors in [12] presented a consensus algorithm with delays and dynamically changing topologies and used the time-domain and frequency-domain approaches to find the stability conditions. Average consensus was considered in [13], where the delays were assumed nonuniform and the communication topology was assumed jointly-connected. The similar problem was discussed in [14] where the communication topology was extended from an undirected graph to a directed one. A second-order consensus regulation algorithm with nonuniform communication delays was studied in [15] with the focus on a flocking problem of large scale multiagent systems. Both delay-independent and delay-dependent conditions were obtained.

The research on the cooperative attitude control problem in the presence of communication delays and dynamically changing topologies was given in $[16,17]$, where a synchronization variable was used to contain both attitude and angular velocity information. Motivated by the work of [18], the conditions to guarantee cooperative attitude control with communication delays were obtained. Similar problem was discussed in [19] with an emphasis on multiple networked Lagrangian systems. Delays, limited data rates, and bounded disturbance input were considered together in the control law.

This paper is organized as follows. In Sections 2 and 3, we provide basics for spacecraft attitude dynamics, graph theory, and functional differential equation. In Section 4, cooperative attitude regulation and tracking problems are described and the control torques with communication delays and dynamically changing topologies are proposed. The case of cooperative attitude regulation with multiple time-varying communication delays and fixed topology is discussed in Section 5, while the case of cooperative attitude tracking with multiple time-varying communication delays and dynamically changing topologies is discussed in Section 6. Simulation results are given in Section 7 to validate the theoretical results. Section 8 contains our concluding remarks.

\section{Preliminaries}

\subsection{Notations}

$\mathbb{R}$ and $\mathbb{C}$ are, respectively, the set of real numbers and the set of complex numbers. $\lambda_{\min }(A)$ and $\lambda_{\max }(A)$ are, respectively, the minimal eigenvalue and the maximum eigenvalue of the matrix $A$. $\otimes$ denotes the Kronecker product. 
Consistent with [20], we denote $\mathbb{C}_{n, \tau}$ as the Banach space of continuous vector functions mapping the interval $[-\tau, 0]$ into $\mathbb{R}^{n}$ with the topology of uniform convergence. $\mathbb{C}_{n, \tau}^{v}=\left\{\phi \in \mathbb{C}_{n, \tau}:\|\phi\|_{c}<v\right\}$, where $v$ is a positive real number.

$\|\cdot\|$ stands for the Euclidean vector norm and $\|\phi\|_{c}=\sup _{-\tau \leq t \leq 0}\|\phi(t)\|$ stands for the norm of a function $\phi \in \mathbb{C}_{n, \tau}$.

$Q>0$ means that the matrix $Q$ is positive definite.

\subsection{Spacecraft Attitude Kinematics and Dynamics}

In this paper, the attitude of each spacecraft in a formation is represented by the unit quaternion, given by $q_{i}=\left[q_{1}, q_{2}, q_{3}, q_{4}\right]_{i}^{T}=\left[\widehat{q}_{i}^{T}, \bar{q}_{i}\right]^{T}, i=1, \ldots, n$. Here $\widehat{q}_{i}=e_{i} \sin \left(\phi_{i} / 2\right)$, $\bar{q}_{i}=\cos \left(\phi_{i} / 2\right)$, where $e_{i}$ and $\phi_{i}$ are the principle axis and the principle angle of the attitude of the $i$ th spacecraft and $q_{i}^{T} q_{i}=1$ [21]. The product of two unit quaternions $p_{i}$ and $q_{i}$ is defined by

$$
q_{i} p_{i}=\left[\begin{array}{c}
\bar{q}_{i} \widehat{p}_{i}+\bar{p}_{i} \widehat{q}_{i}+\widehat{q}_{i} \times \widehat{p}_{i} \\
\bar{q}_{i} \bar{p}_{i}-\widehat{q}_{i}^{T} \widehat{p}_{i}
\end{array}\right] .
$$

The conjugate of the unit quaternion $q_{i}$ is defined by $q_{i}^{-1}=\left[-\widehat{q}_{i}^{T}, \bar{q}_{i}\right]^{T}$. Attitude kinematics and dynamics of each spacecraft using the unit quaternion are given by [21]

$$
\begin{gathered}
\dot{q}_{i}=\frac{1}{2} E_{i}\left(q_{i}\right) \omega_{i}, \quad i=1, \ldots, n, \\
J_{i} \dot{\omega}_{i}=-\omega_{i} \times\left(J_{i} \omega_{i}\right)+\tau_{i}, \quad i=1, \ldots, n,
\end{gathered}
$$

where $q_{i} \in \mathbb{R}^{4}$ denotes the rotation from the body frame of the $i$ th spacecraft to the inertial frame, $\omega_{i}$ is the angular velocity of the $i$ th spacecraft with respect to the inertial frame expressed in the body frame of the $i$ th spacecraft, and $E_{i}\left(q_{i}\right)$ is given by

$$
E_{i}\left(q_{i}\right)=\left[\begin{array}{c}
\bar{q}_{i} I_{3}+S\left(\widehat{q}_{i}\right) \\
-\widehat{q}_{i}^{T}
\end{array}\right]
$$

where $I_{3}$ is the $3 \times 3$ identity matrix, $S(\cdot)$ denotes a $3 \times 3$ skew-symmetric matrix, and $J_{i} \in \mathbb{R}^{3 \times 3}$ and $\tau_{i} \in \mathbb{R}^{3}$ are, respectively, the inertia tensor and control torque of the $i$ th spacecraft.

\subsection{Graph Theory [22]}

The communication topology among spacecraft in the formation is modeled using graph theory. An undirected graph $\mathcal{G}$ consists of a pair $(V, \varepsilon)$, where $V=\left\{v_{1}, \ldots, v_{n}\right\}$ is a finite nonempty set of nodes and $\varepsilon \subseteq V \times V$ is a set of unordered pairs of nodes. An edge $\left(v_{i}, v_{j}\right)$ denotes that nodes $v_{i}$ and $v_{j}$ can obtain information from each other. In such case, nodes $v_{i}$ and $v_{j}$ are neighbors of each other. All the neighbors of node $v_{i}$ are denoted as $N_{i}:=\left\{v_{j} \mid\right.$ $\left.\left(v_{j}, v_{i}\right) \in \mathcal{E}\right\}$, where we assume that $v_{i} \notin N_{i}$.

An undirected path is a sequence of edges in a undirected graph of the form $\left(v_{i_{1}}, v_{i_{2}}\right),\left(v_{i_{2}}, v_{i_{3}}\right), \ldots$. An undirected graph is connected if there is an undirected path between 
every pair of distinct nodes. In this paper, the communication topology is assumed to be undirected.

The adjacency matrix $\mathcal{A}=\left[a_{i j}\right] \in \mathbb{R}^{n \times n}$ associated with the undirected graph $\mathcal{G}$ is defined such that $a_{i j}$ is a positive value if $\left(v_{j}, v_{i}\right) \in \mathcal{E}$, and $a_{i j}=0$ otherwise. We assume that $a_{i j}=a_{j i}$, for all $i \neq j$, since $\left(v_{j}, v_{i}\right) \in \mathcal{E}$ implies $\left(v_{i}, v_{j}\right) \in \mathcal{E}$ in the undirected graph. Also, the Laplacian matrix $\mathcal{L}=\left[l_{i j}\right] \in \mathbb{R}^{n \times n}$ associated with $\mathcal{A}$ is defined as

$$
l_{i j}= \begin{cases}\sum_{j \in N_{i}} a_{i j}, & i=j, \\ -a_{i j}, & i \neq j .\end{cases}
$$

\section{Definitions and Lemmas}

Suppose $f: \mathbb{R} \times \mathbb{C}_{n, \tau}^{v} \mapsto \mathbb{R}^{n}$ is continuous and consider retarded functional differential equation (RFDE)

$$
\dot{x}(t)=f\left(t, x_{t}\right) .
$$

Let $\phi=x_{t}$ be defined as $x_{t}(\theta)=x(t+\theta), \theta \in[-\tau, 0]$. Suppose that the initial condition satisfies $x(\theta)=0$, for all $\theta \in\left[t_{0}-\tau, t_{0}\right]$. Also suppose that the solution $x\left(t_{0}, \phi\right)(t)$ through $\left(t_{0}, \phi\right)$ is continuous in $\left(t_{0}, \phi, t\right)$ in the domain of definition of the function, where $t_{0} \in \mathbb{R}$.

Definition 3.1 (see [23]). The solutions $x\left(t_{0}, \phi\right)$ of the RFDE (3.1) are uniformly asymptotically stable if

(i) for every $\kappa>0$ and for every $t_{0} \geq 0$ there exists a $\delta=\delta(\kappa)$ independent of $t_{0}$ such that for any $\phi \in \mathbb{C}_{n, \tau}^{\delta}$ the solutions $x\left(t_{0}, \phi\right)$ of the RFDE (3.1) satisfies $x_{t}\left(t_{0}, \phi\right) \in \mathbb{C}_{n, \tau}^{\kappa}$ for all $t \geq t_{0}$,

(ii) for every $\eta>0$ and for every $t_{0} \geq 0$ there exists a $T(\eta)$ independent of $t_{0}$ and a $v_{0}>0$ independent of $\eta$ and $t_{0}$ such that for any $\phi \in \mathbb{C}_{n, \tau},\|\phi\|_{c}<v_{0}$ implies that $\left\|x_{t}\left(t_{0}, \phi\right)\right\|_{c}<\eta$, for all $t \geq t_{0}+T(\eta)$.

Definition 3.2 (see [23]). The solutions $x\left(t_{0}, \phi\right)$ of the RFDE (3.1) are uniformly ultimately bounded if there is a $\beta>0$ such that for any $\alpha>0$, there is a constant $T_{0}(\alpha)>0$ such that $\left|x\left(t_{0}, \phi\right)(t)\right| \leq \beta$ for $t \geq t_{0}+T_{0}(\alpha)$ for all $t_{0} \in \mathbb{R}, \phi \in \mathbb{C}$, and $|\phi| \leq \alpha$.

Lemma 3.3 (Lyapunov-Krasovskii stability theorem [20]). Consider the RFDE (3.1). Suppose $f: \mathbb{R} \times \mathbb{C}_{n, \tau} \mapsto \mathbb{R}^{n}$ takes $\mathbb{R} \times$ (bounded sets of $\mathbb{C}_{n, \tau}$ ) into bounded sets of $\mathbb{R}^{n}, u(s), v(s)$, and $w(s)$ are continuous, nonnegative and nondecreasing functions with $u(s), v(s)>0$ for $s \neq 0$ and $u(0)=$ $v(0)=0$. If there exists a continuous function $V: \mathbb{R} \times \mathbb{C}_{n, \tau} \mapsto \mathbb{R}$ such that

(i) $u(\|\phi(0)\|) \leq V(t, \phi) \leq v\left(\|\phi\|_{c}\right)$,

(ii) $\dot{V}(t, \phi) \leq-w(\|\phi(0)\|)$,

then the solutions of (3.1) are uniformly stable. In addition, if $w(s)>0$ for $s>0$, then the solutions of (3.1) are uniformly asymptotically stable. 
Lemma 3.4 (Lyapunov-Razumikhin uniformly ultimately bounded theorem [23]). Consider the RFDE (3.1). Suppose $f: \mathbb{R} \times \mathbb{C}_{n, \tau} \mapsto \mathbb{R}^{n}$ takes $\mathbb{R} \times$ (bounded sets of $\mathbb{C}_{n, \tau}$ ) into bounded sets of $\mathbb{R}^{n}$ and $u, v, w: \mathbb{R}^{+} \mapsto \mathbb{R}^{+}$are continuous nonincreasing functions, $u(s) \rightarrow \infty$ as $s \rightarrow \infty$. If there is a continuous function $V: \mathbb{R} \times \mathbb{R}^{n} \mapsto \mathbb{R}$, a continuous nondecreasing function $p: \mathbb{R}^{+} \mapsto \mathbb{R}^{+}$, $p(s)>s$ for $s>0$, and a constant $H \geq 0$ such that $u(\|x\|) \leq V(x) \leq v(\|x\|), t \in \mathbb{R}, x \in \mathbb{R}^{n}$, and $\dot{V}(t, \phi) \leq-w(\|\phi(0)\|)$ if $\|\phi(0)\| \geq H, V(t+\theta, \phi(\theta))<p(V(t, \phi(0))), \theta \in[-\tau, 0]$, then the solutions of (3.1) are uniformly ultimately bounded.

\section{Problem Statement}

In this paper, we consider cooperative attitude regulation and tracking problems for multiple rigid bodies in the presence of multiple time-varying delays and dynamically changing topologies. The objectives are to guarantee that each spacecraft tracks the constant or timevarying states of the leader spacecraft while aligning their attitudes within the formation. Cooperative attitude regulation control law with zero delay and fixed topology is proposed in $[8]$ as

$$
\tau_{i}=-K_{i} \widehat{q}_{i}-D_{i} \omega_{i}-\sum_{j=1}^{n} a_{i j} \widehat{q}_{i j}-\sum_{j=1}^{n} a_{i j} \omega_{i j},
$$

where $K_{i}$ and $D_{i}$ are nonnegative constants, $a_{i j}$ is the $(i, j)$ th entry of the adjacency matrix A associated with the graph $\mathcal{G}_{1}, q_{i j}=q_{j}^{-1} q_{i}, \widehat{q}_{i j}$ is the vector part of $q_{i j}, \omega_{i j}=\omega_{i}-A\left(q_{i j}\right) \omega_{j}$, and $A\left(q_{i j}\right)=A\left(q_{i}\right) A^{T}\left(q_{j}\right)$ denotes the rotation matrix [21]. Here $q_{i j}$ represents the relative attitude between spacecraft $i$ and spacecraft $j$, and $\omega_{i j}$ represents the relative angular velocity between spacecraft $i$ and spacecraft $j$. Note that the existence of the attitude consensus terms $-\sum_{j=1}^{n} a_{i j} \widehat{q}_{i j}-\sum_{j=1}^{n} a_{i j} \omega_{i j}$ help to guarantee that the attitude of each follower spacecraft will be close to its neighbors. This is necessary in certain spacecraft mission, such as distributed synthetic-aperture imaging mission [24], where the attitude control system is required to have the ability to ensure relative attitude keeping during the maneuver.

Cooperative attitude tracking control law with zero delay and fixed topology is proposed in [9] as

$$
\tau_{i}=J_{i} A\left(\Delta q_{i}\right) \dot{\omega}_{d}+\omega_{d}^{b_{i}} \times J_{i} \omega_{d}^{b_{i}}-K_{i} \widehat{\Delta q}_{i}-D_{i} \Delta \omega_{i}-\sum_{j=1}^{n} a_{i j} \widehat{q}_{i j}-\sum_{j=1}^{n} a_{i j} \omega_{i j}
$$

where $q_{d}$ and $\omega_{d}$ denote, respectively, the time-varyingly desired attitude and angular velocity of the leader spacecraft, $\Delta q_{i}=q_{d}^{-1} q_{i}, \widehat{\Delta q}_{i}$ is the vector part of $\Delta q_{i}, \omega_{d}^{b_{i}}=A\left(\Delta q_{i}\right) \omega_{d}$, $A\left(\Delta q_{i}\right)=A\left(q_{i}\right) A^{T}\left(q_{d}\right)$, and $\Delta \omega_{i}=\omega_{i}-\omega_{d}^{b_{i}}$. Here $\Delta q_{i}$ denotes the relative attitude between spacecraft $i$ and the leader, $\Delta \omega_{i}$ denotes the relative angular velocity between spacecraft $i$ and the leader. By using (4.1) for (2.2), cooperative attitude regulation, that is, $q_{i} \rightarrow q_{I}$ and $\omega_{i} \rightarrow 0$, is achieved, where $q_{I}$ denotes the identity quaternion $[0,0,0,1]^{T}$. By using (4.2) for (2.2), cooperative attitude tracking, that is, $q_{i} \rightarrow q_{d}$ and $\omega_{i} \rightarrow \omega_{d}$ is achieved.

In this paper, we extend cooperative attitude regulation and tracking control laws to the cases where there exist multiple time-varying communication delays and dynamically changing topologies. For the first part, we discuss cooperative attitude regulation problem 
in the presence of multiple time-varying communication delays and assume that the communication topology is fixed. A model-independent control torque $\tau_{i}$ is proposed

$$
\tau_{i}=-K_{i} \widehat{q}_{i}-D_{i} \omega_{i}-\sum_{j=1}^{n} a_{i j}\left[\widehat{q}_{i}-\widehat{q}_{j}\left(t-T_{i j}(t)\right)\right]-\sum_{j=1}^{n} a_{i j}\left[\omega_{i}-l_{i j} A_{i j}\left(t-T_{i j}(t)\right) \omega_{j}\left(t-T_{i j}(t)\right)\right]
$$

where $K_{i}, D_{i}$, and $a_{i j}$ are defined after (4.1), $l_{i j}$ is a nonnegative constant, $T_{i j}=T_{j i}$ denotes multiple time-varying communication delay, and $A_{i j}\left(t-T_{i j}(t)\right)=A\left(q_{i}\right) A^{T}\left(q_{j}\left(t-T_{i j}\right)\right)$. For the second part, we discuss cooperative attitude tracking problem in the presence of multiple time-varying communication delays and dynamically changing topologies, where a modelindependent control torque $\tau_{i}$ is proposed,

$$
\tau_{i}=-K_{i} \widehat{\Delta q}_{i}-D_{i} \Delta \omega_{i}-\sum_{j=1}^{n} a_{i j}^{\sigma}\left[\widehat{\Delta q}_{i}-\widehat{\Delta q}_{j}\left(t-T_{i j}(t)\right)\right]
$$

where $\widehat{\Delta q_{i}}$ and $\Delta \omega_{i}$ are defined after (4.2). Following the similar definition given in [25], the dynamically changing topology is defined as $\sigma:[0,+\infty) \rightarrow \psi_{\Gamma}$, where the set $\Gamma$ is a finite collection of undirected graphs with a common node set. Then $a_{i j}^{\sigma}$ denotes the $(i, j)$ th entry of the adjacency matrix $\mathcal{A}_{\sigma}$ associated with the communication topology $\mathcal{G}_{\sigma}$. Before moving on, we assume that $\omega_{d}$ and $\dot{\omega}_{d}$ are bounded and define $\gamma_{J_{i}}=\left\|J_{i}\right\|, \gamma_{d}=\sup _{t \geq 0}\left\|\omega_{d}(t)\right\|$, and $\beta_{1}=\left\|\dot{\omega}_{d}\right\|+\left\|\omega_{d}\right\|^{2}$ in this paper.

Remark 4.1. Compared with (4.3), (4.4) introduces absolute angular velocity damping, thus avoiding introducing the communication delays of relative angular velocity information between the follower spacecraft.

\section{Cooperative Attitude Regulation with Multiple Time-Varying Communication Delays and Fixed Topology}

In this section, we propose proper conditions to guarantee that cooperative attitude regulation is achieved by using (4.3) for (2.2). Before moving on, we need the following lemma.

Lemma 5.1. The matrix $M=\mathcal{L}+\operatorname{diag}\left(K_{1}, \ldots, K_{n}\right)$ is symmetric and positive definite if the undirected graph $\mathcal{G}$ is connected and at least one $K_{i}>0$, where $\mathcal{\perp}$ is the Laplacian matrix of graph $\mathcal{G}$.

Proof. See the proof of Lemma 4 [25].

Motivated by the works of $[15,16,18]$, we provide the following theorem for closedloop systems (2.2) with (4.3). 
Theorem 5.2. Using (4.3) for (2.2), if $M>0, D_{i}>0$, for all $i, \rho_{i j}<a_{i j}, 4 \rho_{i j}\left(1-\dot{T}_{i j}\right)\left(a_{i j}-\rho_{i j}\right)>$ $l_{i j}^{2} a_{i j}^{2}$, when $a_{i j} \neq 0$, and $W>0$, cooperative attitude regulation, that is, $q_{i} \rightarrow q_{I}$ and $\omega_{i} \rightarrow 0$, is achieved, where $W$ is given by

$$
W=\left[\begin{array}{cc}
c M-c^{2} \boldsymbol{\Phi}-\frac{1}{2} c^{2} \boldsymbol{B} & \frac{(M-K-c \mathcal{B}-c \mathcal{C})}{2} \\
\frac{(M-K-c \mathcal{B}-c \mathcal{C})}{2} & D-B-\frac{1}{2} c \mathcal{F}
\end{array}\right]
$$

and $c, \rho_{i j}, D, K, M, B, \mathcal{C}, \boldsymbol{D}$, and $\mathcal{F}$ are defined in the proof.

Proof. Consider the following Lyapunov function candidate

$$
\begin{aligned}
V= & \sum_{i=1}^{n}\left(K_{i}+c D_{i}\right)\left\|q_{i}-q_{I}\right\|^{2}+\frac{1}{2} \sum_{i=1}^{n} \omega_{i}^{T} J_{i} \omega_{i}+\sum_{i=1}^{n} c \widehat{q}_{i}^{T} J_{i} \omega_{i}+\sum_{i=1}^{n} \sum_{j=1}^{n} \int_{-T_{i j}(t)}^{0} \rho_{i j}\left\|\omega_{j}(t+s)\right\|^{2} d s \\
& +\frac{1}{2} \sum_{i=1}^{n} \sum_{j=1}^{n} \int_{-T_{i j}(t)}^{0} \int_{\mu}^{0} a_{i j}\left\|\hat{\widehat{q}}_{j}(t+s)\right\|^{2} d s d \mu,
\end{aligned}
$$

where $c$ is a positive constant, $\rho_{i j}=0$ when $a_{i j}=0$, and $\rho_{i j}$ is a positive constant when $a_{i j} \neq 0$. It is easy to verify that $V$ is positive definite if $2\left(K_{i}+c D_{i}\right) \lambda_{\min }\left(J_{i}\right)>c^{2} \lambda_{\max }^{2}\left(J_{i}\right)$, for all $i$ [26]. This implies that the selection of sufficiently small $c$ guarantees that $V$ is positive definite. Taking the derivative of $V$ gives

$$
\begin{aligned}
\dot{V}= & \sum_{i=1}^{n}\left(K_{i}+c D_{i}\right) \widehat{q}_{i}^{T} \omega_{i}-\sum_{i=1}^{n}\left(\omega_{i}+c \widehat{q}_{i}\right)^{T}\left[K_{i} \widehat{q}_{i}+\sum_{j=1}^{n} a_{i j}\left(\widehat{q}_{i}(t)-\widehat{q}_{j}(t)\right)\right] \\
& -\sum_{i=1}^{n}\left(\omega_{i}+c \widehat{q}_{i}\right)^{T}\left\{D_{i} \omega_{i}+\sum_{j=1}^{n} a_{i j}\left[\omega_{i}-l_{i j} A_{i j}\left(t-T_{i j}(t)\right) \omega_{j}\left(t-T_{i j}(t)\right)\right]\right\}+\sum_{i=1}^{n} c \widehat{q}_{i}^{T}\left(-\omega_{i} \times J_{i} \omega_{i}\right) \\
& +\frac{1}{2} \sum_{i=1}^{n} c\left[\left(\bar{q}_{i} I_{3}+S\left(\widehat{q}_{i}\right)\right) \omega_{i}\right]^{T} J_{i} \omega_{i}-\sum_{i=1}^{n}\left(\omega_{i}+c \widehat{q}_{i}\right)^{T} \sum_{j=1}^{n} \int_{-T_{i j}(t)}^{0} a_{i j} \dot{\hat{q}}_{j}(t+\mu) d \mu \\
& +\frac{1}{2} \sum_{i=1}^{n} \sum_{j=1}^{n} \int_{-T_{i j}(t)}^{0} a_{i j}\left[\hat{\widehat{q}}_{j}^{T}(t) \dot{\widehat{q}}_{j}(t)-\dot{\hat{q}}_{j}(t+\mu)^{T} \dot{\hat{q}}_{j}(t+\mu)\right] d \mu \\
& +\rho_{i j} \sum_{i=1}^{n} \sum_{j=1}^{n} \omega_{j}(t)^{T} \omega_{j}(t)-\rho_{i j} \sum_{i=1}^{n} \sum_{j=1}^{n}\left(1-\dot{T}_{i j}\right) \omega_{j}\left(t-T_{i j}\right)^{T} \omega_{j}\left(t-T_{i j}\right),
\end{aligned}
$$


where we have used the fact that $\omega_{i}^{T}\left(\omega_{i} \times J_{i} \omega_{i}\right)=0$ and Leibniz-Newton formula $\widehat{q}_{j}\left(t-T_{i j}(t)\right)=$ $\widehat{q}_{j}(t)-\int_{-T_{i j}}^{0} \dot{\hat{q}}_{j}(t+\mu) d \mu[23]$. It thus follows that

$$
\begin{aligned}
\dot{V} \leq & \frac{1}{2} c \sum_{i=1}^{n} \gamma_{J_{i}}\left\|\omega_{i}\right\|^{2}-c \widehat{q}^{T}\left(M \otimes I_{3}\right) \widehat{q}-\omega^{T}\left(M \otimes I_{3}\right) \widehat{q}+\widehat{q}^{T}(K+c D) \otimes I_{3} \omega \\
& +\frac{1}{2} \sum_{i=1}^{n} \sum_{j=1}^{n} T_{i j} a_{i j}\left\|\omega_{i}+c \widehat{q}_{i}\right\|^{2} \\
& -\frac{1}{2} \sum_{i=1}^{n} \sum_{j=1}^{n} \int_{-T_{i j}}^{0} a_{i j}\left\|\omega_{i}+c \widehat{q}_{i}+\dot{\hat{q}}_{j}(t+\mu)\right\|^{2} d \mu \\
& +\frac{1}{2} \sum_{i=1}^{n} \sum_{j=1}^{n} T_{i j} a_{i j} \omega_{j}^{T}\left(\bar{q}_{j} I_{3}+S\left(\widehat{q}_{j}\right)\right)^{T}\left(\bar{q}_{j} I_{3}+S\left(\widehat{q}_{j}\right)\right) \omega_{j} \\
& -\sum_{i=1}^{n} \sum_{j=1}^{n}\left(a_{i j}-\rho_{i j}\right)\left\|\left(\omega_{i}+c \widehat{q}_{i}\right)-\frac{l_{i j} a_{i j}}{2\left(a_{i j}-\rho_{i j}\right)} A_{i j}\left(t-T_{i j}\right) \omega_{j}\left(t-T_{i j}\right)\right\|^{2} \\
& +\rho_{i j} \sum_{i=1}^{n} \sum_{j=1}^{n} \omega_{j}^{T} \omega_{j}+\sum_{i=1}^{n} \sum_{j=1}^{n}\left(a_{i j}-\rho_{i j}\right)\left\|\omega_{i}+c \widehat{q}_{i}\right\|^{2} \\
& -\sum_{i=1}^{n} \sum_{j=1}^{n} a_{i j}\left(\omega_{i}+c \widehat{q}_{i}\right)^{T} \omega_{i}-\sum_{i=1}^{n} \sum_{j=1}^{n}\left[\rho_{i j}\left(1-\dot{T}_{i j}\right)-\frac{l_{i j}^{2} a_{i j}^{2}}{4\left(a_{i j}-\rho_{i j}\right)}\right]\left\|\omega_{i}\left(t-T_{i j}\right)\right\|^{2} \\
& -\sum_{i=1}^{n} D_{i}\left\|\omega_{i}\right\|^{2}-\sum_{i=1}^{n} c D_{i} \widehat{q}_{i}^{T} \omega_{i}
\end{aligned}
$$

where $\widehat{q}=\left[\hat{q}_{1}^{T}, \widehat{q}_{2}^{T}, \ldots, \widehat{q}_{n}^{T}\right]^{T}, \omega=\left[\omega_{1}^{T}, \omega_{2}^{T}, \ldots, \omega_{n}^{T}\right]^{T}, K=\operatorname{diag}\left(K_{1}, \ldots, K_{n}\right), M=\mathcal{L}+K$, and $D=\operatorname{diag}\left(D_{1}, \ldots, D_{n}\right)$. Based on the conditions that $\rho_{i j}<a_{i j}$ and $4 \rho_{i j}\left(1-\dot{T}_{i j}\right)\left(a_{i j}-\rho_{i j}\right)>l_{i j}^{2} a_{i j}^{2}$ when $a_{i j} \neq 0$, we have that

$$
\begin{aligned}
\dot{V} \leq & \frac{1}{2} c \sum_{i=1}^{n} \gamma_{J_{i}}\left\|\omega_{i}\right\|^{2}-c \widehat{q}^{T}\left(M \otimes I_{3}\right) \widehat{q}-\omega^{T}\left(M \otimes I_{3}\right) \widehat{q}+\widehat{q}^{T}\left(K \otimes I_{3}\right) \omega \\
& -\omega^{T}\left(D \otimes I_{3}\right) \omega+\frac{1}{2} \sum_{i=1}^{n} \sum_{j=1}^{n} T_{i j} a_{i j}\left\|\omega_{i}+c \widehat{q}_{i}\right\|^{2} \\
& +\frac{1}{2} \sum_{i=1}^{n} \sum_{j=1}^{n} T_{i j} a_{i j} \omega_{i}^{T} \omega_{i}+c \sum_{i=1}^{n} \sum_{j=1}^{n}\left(a_{i j}-2 \rho_{i j}\right) \omega_{i}^{T} \widehat{q}_{i}+c^{2} \sum_{i=1}^{n} \sum_{j=1}^{n}\left(a_{i j}-\rho_{i j}\right) \widehat{q}_{i}^{T} \widehat{q}_{i} \\
= & -\widehat{q}^{T}\left(c M-c^{2} \Phi-\frac{1}{2} c^{2} 乃\right) \otimes I_{3} \widehat{q}+\omega^{T}(-M+K+c \mathcal{B}+c \mathcal{C}) \\
& \otimes I_{3} \widehat{q}-\omega^{T}\left(D-B-\frac{1}{2} c \mathcal{F}\right) \otimes I_{3} \omega
\end{aligned}
$$


where we have used the fact that $\sum_{i=1}^{n} \sum_{j=1}^{n} a_{i j} \omega_{j}^{T} \omega_{j}=\sum_{i=1}^{n} \sum_{j=1}^{n} a_{i j} \omega_{i}^{T} \omega_{i}$ (graph $\mathcal{G}$ is undirected). Here we also define $\boldsymbol{B}=\left[b_{i j}\right], \mathcal{C}=\left[c_{i j}\right], \boldsymbol{D}=\left[d_{i j}\right]$, and $\mathcal{F}$ as $n \times n$ matrices, where $b_{i j}=T_{i j} a_{i j}, c_{i j}=a_{i j}-2 \rho_{i j}, d_{i j}=a_{i j}-\rho_{i j}$, and $\mathcal{F}=\operatorname{diag}\left(\gamma_{J_{1}}, \ldots, \gamma_{J_{n}}\right)$. Based on the conditions that $M$ is positive definite and $D$ is positive definite $\left(D_{i}>0\right.$, for all $\left.i\right)$, for the sufficient small $T_{i j}$ and $c$, it is easy to verify that there always exist $M$ and $D$ to guarantee $W$ is positive definite. Then, Lemma 3.3 implies the stability of the closed-loop systems (2.2) with (4.3) from the condition that $W>0$. Thus, cooperative attitude regulation, that is, $q_{i} \rightarrow q_{I}$, and $\omega_{i} \rightarrow 0$ is achieved under the conditions provided in Theorem 5.2.

Remark 5.3. It follows that $M$ is positive definite from Lemma 5.1 if the undirected graph $\mathcal{G}$ is connected and at least one $K_{i}>0$. This implies conditions that the undirected graph $\mathcal{G}$ is connected and at least one $K_{i}>0$ can be used to replace condition that $M>0$.

Remark 5.4. Note that the parameters $\rho_{i j}$ and $c$ in the proposed conditions in Theorem 5.2 are independent of control parameters in control torque (4.3).

Remark 5.5. The cooperative attitude regulation problem in the presence of communication delays was also discussed in the work of [16]. In contrast to [16], we do not assume that relative attitude information and relative angular velocity information between different follower spacecraft could be described in a united variable. This may increase the flexibility of the design.

\section{Cooperative Attitude Tracking with Multiple Time-Varying Communication Delays and Dynamically Changing Topologies}

In this section, the conditions to guarantee cooperative attitude tracking in the presence of multiple time-varying communication delays and dynamically changing topologies are obtained. We first transform the closed-loop systems (2.2) to the error kinematic and dynamic as

$$
\begin{gathered}
\dot{\Delta} q_{i}=\frac{1}{2} E_{i}\left(\Delta q_{i}\right) \Delta \omega_{i} \\
J_{i} \dot{\Delta} \omega_{i}=-\omega_{i} \times\left(J_{i} \omega_{i}\right)-J_{i} A\left(\Delta q_{i}\right) \dot{\omega}_{d}+J_{i} \Delta \omega_{i} \times \omega_{d}^{b_{i}}+\tau_{i},
\end{gathered}
$$

where $\Delta q_{i}, \Delta \omega_{i}$, and $\omega_{d}^{b_{i}}$ are defined after (4.2). Also define $\tau=\left[\tau_{1}^{T}, \ldots, \tau_{n}^{T}\right]^{T}$. We can then transform (4.4) to the matrix expression

$$
\tau=-\left(M_{\sigma} \otimes I_{3}\right) \widehat{\Delta q}-\left(D \otimes I_{3}\right) \Delta \omega-\sum_{k=1}^{r}\left(\mathcal{A}_{k}^{\sigma} \otimes I_{3}\right) \int_{-T_{k}(t)}^{0} \widehat{\Delta q}(t+\mu) d \mu,
$$




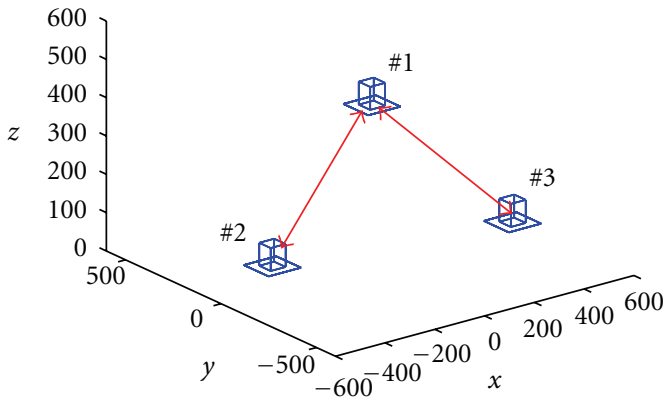

(a) Communication topology $\mathcal{G}_{1}$

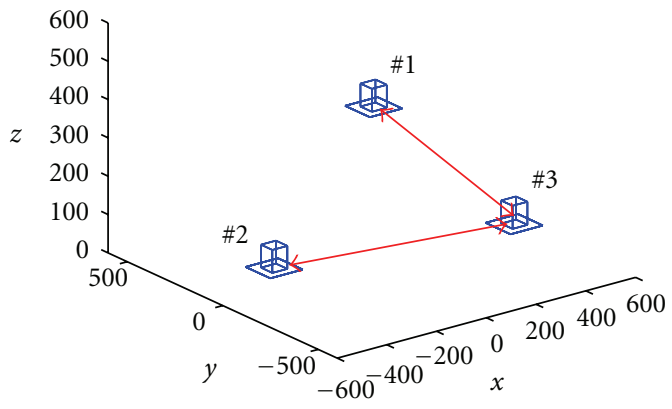

(b) Communication topology $\mathcal{G}_{2}$

Figure 1: Communication topologies.

where $\widehat{\Delta q}=\left[\widehat{\Delta q}_{1}^{T}, \widehat{\Delta q}_{2}^{T}, \ldots, \widehat{\Delta q}_{n}^{T}\right]^{T}, \Delta \omega=\left[\Delta \omega_{1}^{T}, \Delta \omega_{2}^{T}, \ldots, \Delta \omega_{n}^{T}\right]^{T}, K=\operatorname{diag}\left(K_{1}, \ldots, K_{n}\right), M_{\sigma}=$ $L_{\sigma}+K, L_{\sigma}$ is the Laplacian matrix of $\mathcal{G}_{\sigma}, D=\operatorname{diag}\left(D_{1}, \ldots, D_{n}\right), T_{k}(t) \in\left\{T_{i j}(t): i, j=1, \ldots, n\right\}$ for $k=1, \ldots, r$, and $r=n(n-1) / 2, \mathcal{A}_{k}^{\sigma}=\left[a_{i j k}\right]$ is a corresponding $n \times n$ matrix, where

$$
a_{i j k}= \begin{cases}a_{i j}^{\sigma}, & T_{k}=T_{i j}, \\ 0, & T_{k} \neq T_{i j} .\end{cases}
$$

Before moving on, we need the following lemma.

Lemma 6.1 ([27]). For any $a, b \in \mathbb{R}^{n}$ and any symmetric positive definite matrix $\Phi \in \mathbb{R}^{n \times n}$, one has $2 a^{T} b \leq a^{T} \Phi^{-1} a+b^{T} \Phi b$.

Theorem 6.2. Using (4.4) for (2.2), if $M_{\sigma}>0$ in each time interval, $D_{i}>0$, for all $i$, and $Q=\left[\begin{array}{ll}Q_{11} & Q_{12} \\ Q_{12} & Q_{22}\end{array}\right]>0$, where $Q_{11}=c M_{\sigma}-(1 / 2) c^{2} \sum_{k=1}^{r} T_{k} \mathcal{A}_{k}^{\sigma}\left(\mathcal{A}_{k}^{\sigma}\right)^{T}, Q_{12}=\left[-K+M_{\sigma}-\right.$ $\left.c \sum_{k=1}^{r} T_{k} \mathcal{A}_{k}^{\sigma}\left(\mathcal{A}_{k}^{\sigma}\right)^{T}\right] / 2, Q_{22}=D-\mathcal{F}_{\epsilon}-(1 / 2) \sum_{k=1}^{r} T_{k} \mathcal{A}_{k}^{\sigma}\left(\mathcal{A}_{k}^{\sigma}\right)^{T}$, and $\mathcal{F}_{\epsilon}=\operatorname{diag}\left((3 / 2) c \gamma_{J_{1}}+\right.$ $\left.(1 / 2) \sum_{k=1}^{r} T_{k} q\left(\lambda_{\max }(P 2) / \lambda_{\min }(P 1)\right), \ldots,(3 / 2) c \gamma_{J_{n}}+(1 / 2) \sum_{k=1}^{r} T_{k} q\left(\lambda_{\max }(P 2) / \lambda_{\min }(P 1)\right)\right)$, the error state $x$ of the closed-loop system is uniformly ultimately bounded, where $x=\left[\widehat{\Delta q}^{T}, \Delta \omega^{T}\right]^{T}$. In particular, the ultimate bound of $x$ is $\lambda_{\max }(P 2) b / \lambda_{\min }(P 1) \theta \lambda_{\min }(Q)(c, q, P 1, P 2, b, \theta$ will be defined in the proof).

Proof. Consider the following Lyapunov function candidate

$$
V=\sum_{i=1}^{n}\left(K_{i}+c D_{i}\right)\left\|\Delta q_{i}-q_{I}\right\|^{2}+\frac{1}{2} \sum_{i=1}^{n} \Delta \omega_{i}^{T} J_{i} \Delta \omega_{i}+\sum_{i=1}^{n} c \widehat{\Delta q}_{i}^{T} J_{i} \Delta \omega_{i}
$$




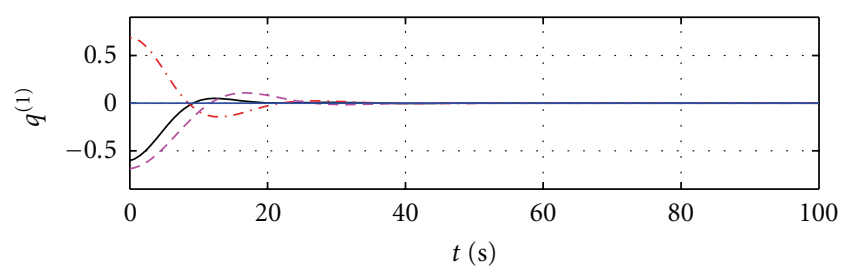

(a)

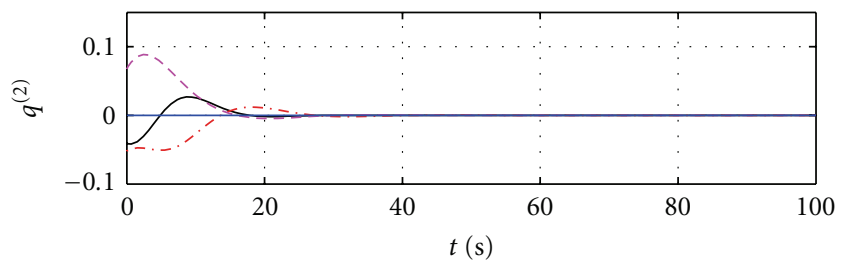

(b)

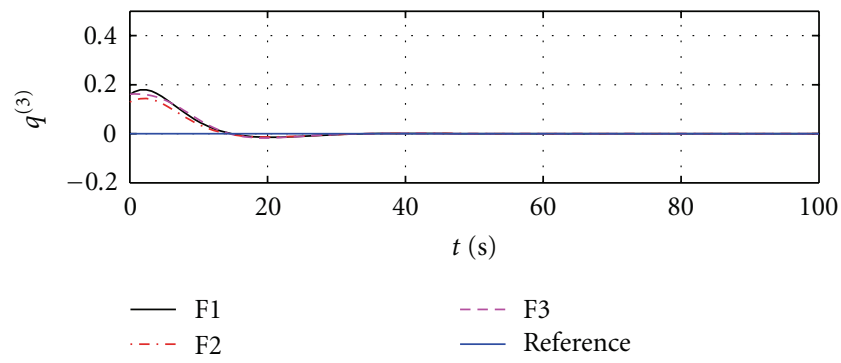

(c)

Figure 2: Rigid body attitudes with control law (4.3).

where $c$ is a positive constant. By using the fact that $\left\|\Delta q_{i}-q_{I}\right\|^{2} \leq 2\left\|\widehat{\Delta q_{i}}\right\|^{2}$, we know that $x^{T} P_{1} x \leq V \leq x^{T} P_{2} x$, where

$$
\begin{gathered}
P_{1}=\left[\begin{array}{cc}
(K+c D) \otimes I_{3} & \frac{1}{2} c J \otimes I_{3} \\
\frac{1}{2} c J \otimes I_{3} & \frac{1}{2} J \otimes I_{3}
\end{array}\right], \\
P_{2}=\left[\begin{array}{cc}
2(K+c D) \otimes I_{3} & \frac{1}{2} c J \otimes I_{3} \\
\frac{1}{2} c J \otimes I_{3} & \frac{1}{2} J \otimes I_{3}
\end{array}\right],
\end{gathered}
$$




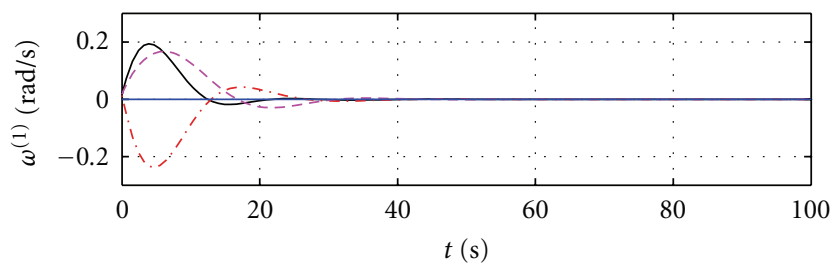

(a)

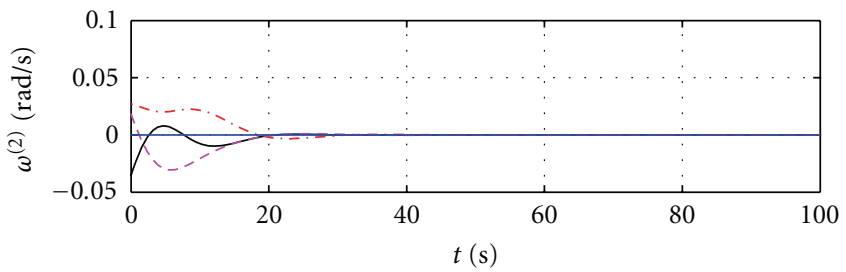

(b)

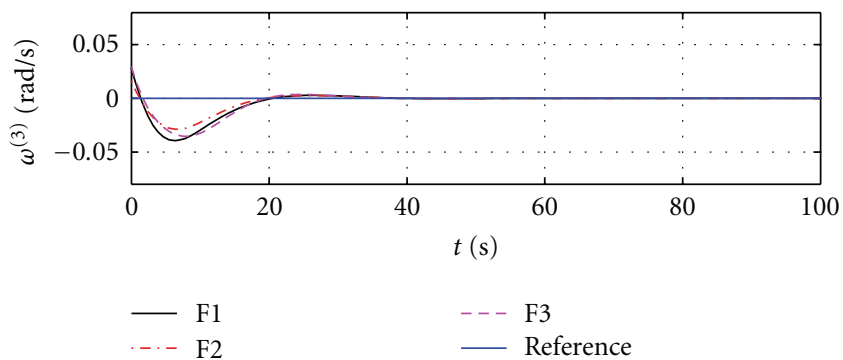

(c)

Figure 3: Rigid body angular velocities with control law (4.3).

and $J=\operatorname{diag}\left(J_{1}, \ldots, J_{n}\right)$. We also know that $V$ is positive definite if $c$ is chosen properly to ensure $2\left(K_{i}+c D_{i}\right) \lambda_{\min }\left(J_{i}\right)>c^{2} \lambda_{\max }^{2}\left(J_{i}\right)$, for all $i$. Taking the derivative of $V$ gives

$$
\begin{aligned}
\dot{V} \leq & \sum_{i=1}^{n}\left(K_{i}+c D_{i}\right) \widehat{\Delta q}_{i}^{T} \Delta \omega_{i}-\sum_{i=1}^{n}\left(\Delta \omega_{i}+c \widehat{\Delta q_{i}}\right)^{T}\left[K_{i} \widehat{\Delta q_{i}}+\sum_{j=1}^{n} a_{i j}^{\sigma}\left(\widehat{\Delta q}_{i}(t)-\widehat{\Delta q_{j}}(t)\right)+D_{i} \Delta \omega_{i}\right] \\
+ & \sum_{i=1}^{n}\left\{\gamma_{J_{i}} \beta_{1}\left\|\Delta \omega_{i}\right\|+c \gamma_{J_{i}} \beta_{1}\left\|\widehat{\Delta q_{i}}\right\|+c \gamma_{J_{i}}\left\|\Delta \omega_{i}\right\|^{2}+3 c \gamma_{J_{i}} \gamma_{J_{d}}\left\|\widehat{\Delta q_{i}}\right\|\left\|\Delta \omega_{i}\right\|\right. \\
& \left.+\frac{1}{2} c\left[\left(\overline{\Delta q}_{i} I_{3}+S\left(\widehat{\Delta q_{i}}\right)\right) \Delta \omega_{i}\right]^{T} J_{i} \Delta \omega_{i}\right\} \\
+ & (\Delta \omega+c \widehat{\Delta q})^{T} \sum_{k=1}^{r}\left(\mathcal{A}_{k}^{\sigma} \otimes I_{3}\right) \int_{-T_{k}(t)}^{0} \widehat{\Delta q}(t+\mu) d \mu,
\end{aligned}
$$

where we have used (6.1), and the facts that $\Delta \omega_{i}^{T}\left(-\omega_{i} \times\left(J_{i} \omega_{i}\right)-J_{i} A\left(\Delta q_{i}\right) \dot{\omega}_{d}+J_{i} \Delta \omega_{i} \times \omega_{d}^{b_{i}}\right)=$ $\Delta \omega_{i}^{T}\left(-J_{i} A\left(\Delta q_{i}\right) \dot{\omega}_{d}-\omega_{d}^{b i} \times J_{i} \omega_{d}^{b i}\right) \leq \gamma_{J_{i}} \beta_{1}\left\|\Delta \omega_{i}\right\|$ and ${\widehat{\Delta q_{i}^{T}}}^{T}\left(-\omega_{i} \times\left(J_{i} \omega_{i}\right)-J_{i} A\left(\Delta q_{i}\right) \dot{\omega}_{d}+J_{i} \Delta \omega_{i} \times \omega_{d}^{b_{i}}\right)=$ 


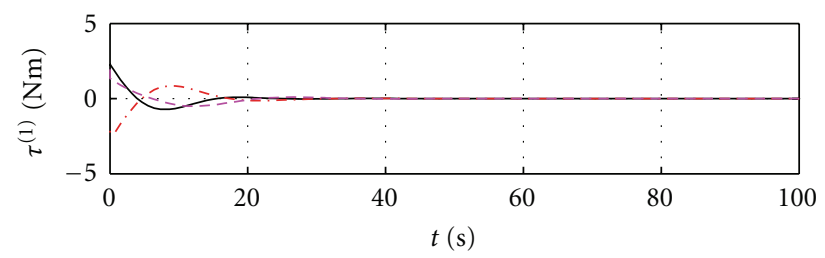

(a)

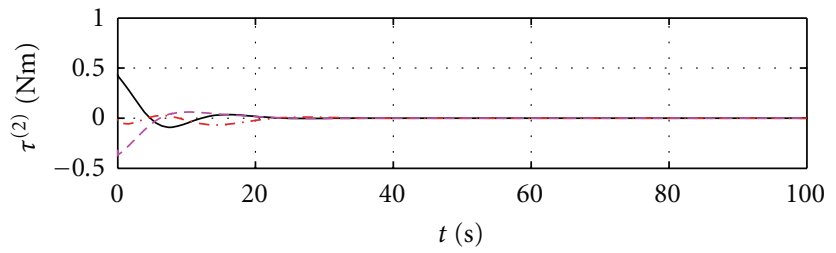

(b)

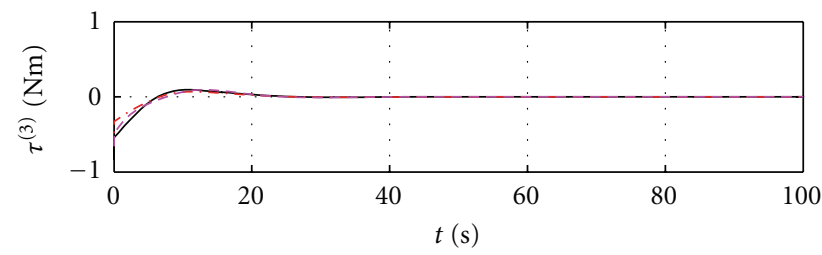

- F1

-.. F2

-- F3

(c)

Figure 4: Rigid body control torques with control law (4.3).

$\widehat{\Delta q}_{i}^{T}\left(-J_{i} A\left(\Delta q_{i}\right) \dot{\omega}_{d}-\omega_{d}^{b i} \times J_{i} \omega_{d}^{b i}+J_{i} \Delta \omega_{i} \times \omega_{d}^{b_{i}}-\omega_{d}^{b_{i}} \times J_{i} \Delta \omega_{i}-\Delta \omega_{i} \times J_{i} \Delta \omega_{i}-\Delta \omega_{i} \times J_{i} \omega_{d}^{b_{i}}\right) \leq$ $\gamma_{J_{i}} \beta_{1}\left\|\widehat{\Delta q_{i}}\right\|+\gamma_{J_{i}}\left\|\Delta \omega_{i}\right\|^{2}+3 \gamma_{J_{i}} \gamma_{d}\left\|\widehat{\Delta q}_{i}\right\|\left\|\Delta \omega_{i}\right\|$. We then have that

$$
\begin{aligned}
\dot{V} \leq & \widehat{\Delta q}^{T}(K+c D) \otimes I_{3} \Delta \omega-c \widehat{\Delta q}^{T}\left(M_{\sigma} \otimes I_{3}\right) \widehat{\Delta q}-\widehat{\Delta q}^{T}\left(M_{\sigma} \otimes I_{3}\right) \Delta \omega \\
& -\Delta \omega^{T}\left(D \otimes I_{3}\right) \Delta \omega-c \widehat{\Delta q}^{T}\left(D \otimes I_{3}\right) \Delta \omega \\
& +\sum_{i=1}^{n} \beta_{1} \gamma_{J_{i}}\left\|\Delta \omega_{i}\right\|+\sum_{i=1}^{n} c \beta_{1} \gamma_{J_{i}}\left\|\widehat{\Delta q_{i}}\right\|+\frac{3}{2} \sum_{i=1}^{n} c \gamma_{J_{i}}\left\|\Delta \omega_{i}\right\|^{2}+\sum_{i=1}^{n} 3 c \gamma_{J_{d}} \gamma_{J_{i}}\left\|\Delta \omega_{i}\right\| \\
& +\frac{1}{2} \sum_{k=1}^{r} \int_{-T_{k}(t)}^{0}(\Delta \omega+c \widehat{\Delta q})^{T}\left[\mathcal{A}_{k}^{\sigma}\left(\mathcal{A}_{k}^{\sigma}\right)^{T}\right] \otimes I_{3}(\Delta \omega+c \widehat{\Delta q}) \\
& +\frac{1}{2} \sum_{k=1}^{r} \int_{-T_{k}(t)}^{0} \widehat{\Delta q}(t+\mu)^{T} \widehat{\Delta q}(t+\mu) d \mu,
\end{aligned}
$$




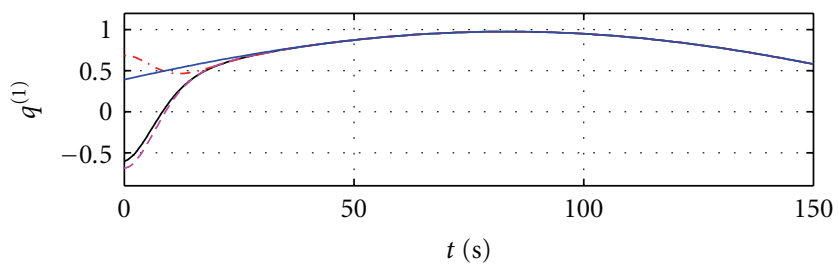

(a)

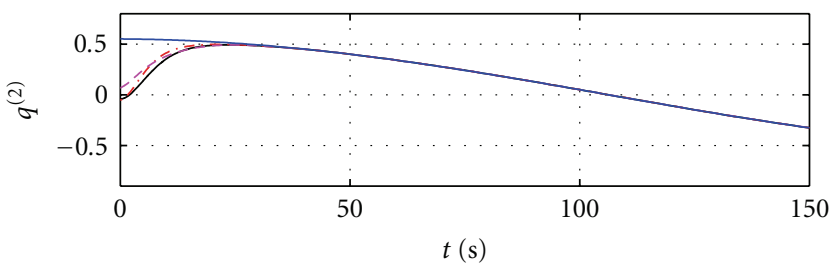

(b)

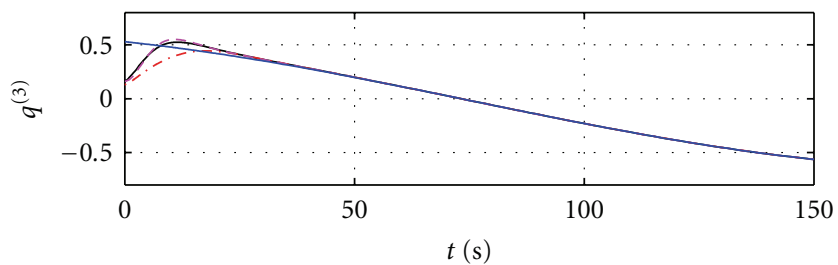

$-\mathrm{F} 1$

- - F3

- Reference

(c)

Figure 5: Rigid body attitudes with control law (4.4).

where we have used the fact that $\left\|\widehat{\Delta q_{i}}\right\| \leq 1$, for all $i$ and Lemma 6.1 to derive the inequality. Take $\phi(s)=q s$ for some constant $q>1$. In the case of

$$
V(x(t+\theta))<q V(x(t)), \quad-\sup _{k}\left\{T_{k}\right\} \leq \theta \leq 0,
$$

we know that $\lambda_{\min }\left(P_{1}\right)\|\Delta \omega\|^{2}(t+\theta)<q \lambda_{\max }\left(P_{2}\right)\left(\|\Delta \omega\|^{2}+\|\widehat{\Delta q}\|^{2}\right)$. (Note that this is a property inherited from Lyapunov-Razumikhin uniformly ultimately bounded theorem.) Thus, we have that

$$
\begin{aligned}
\dot{V} \leq & \widehat{\Delta q}^{T}\left(K-M_{\sigma}\right) \otimes I_{3} \Delta \omega-c \widehat{\Delta q}^{T}\left(M_{\sigma} \otimes I_{3}\right) \widehat{\Delta q}-\Delta \omega^{T}\left(D \otimes I_{3}\right) \Delta \omega \\
& +\sum_{i=1}^{n} \beta_{1} \gamma_{J_{i}}\left\|\Delta \omega_{i}\right\|+\sum_{i=1}^{n} c \beta_{1} \gamma_{J_{i}}\left\|\widehat{\Delta q_{i}}\right\|+\frac{3}{2} \sum_{i=1}^{n} c \gamma_{J_{i}}\left\|\Delta \omega_{i}\right\|^{2}+\sum_{i=1}^{n} 3 c \gamma_{J_{d}} \gamma_{J_{i}}\left\|\Delta \omega_{i}\right\| \\
& +\frac{1}{2} \sum_{k=1}^{r} T_{k}(\Delta \omega+c \widehat{\Delta q})^{T}\left[\mathcal{A}_{k}^{\sigma}\left(\mathcal{A}_{k}^{\sigma}\right)^{T}\right] \otimes I_{3}(\Delta \omega+c \widehat{\Delta q}) \\
& +\frac{1}{2} \sum_{k=1}^{r} T_{k} q \frac{\lambda_{\max }\left(P_{2}\right)}{\lambda_{\min }\left(P_{1}\right)}\left(\|\Delta \omega\|^{2}+\|\widehat{\Delta q}\|^{2}\right)=-x^{T}\left(Q \otimes I_{3}\right) x+w^{T} y,
\end{aligned}
$$




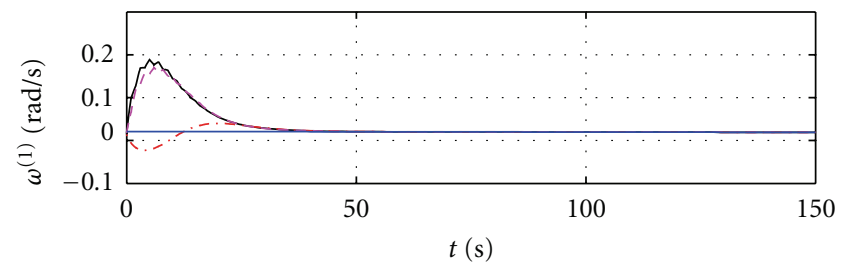

(a)

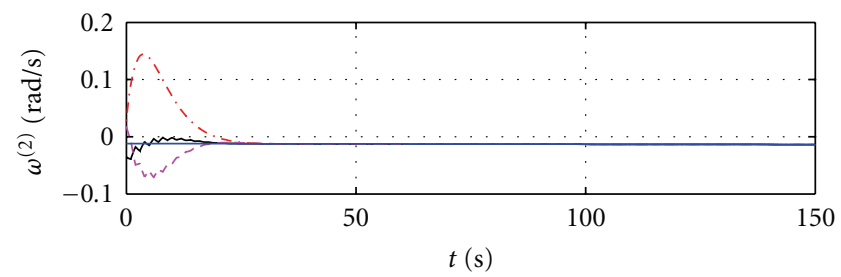

(b)

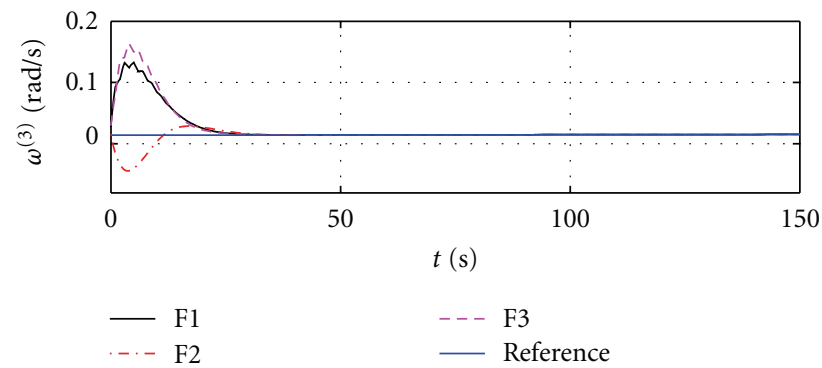

(c)

Figure 6: Rigid body angular velocities with control law (4.4).

where $y=\left[\left\|\widehat{\Delta q}_{1}\right\|, \ldots,\left\|\widehat{\Delta q_{n}}\right\|,\left\|\Delta \omega_{1}\right\|, \ldots,\left\|\Delta \omega_{n}\right\|\right]^{T}, Q$ is defined in Theorem 6.2, and $w=$ $\left[c \beta_{1} \gamma_{J_{1}}+(1 / 2) \sum_{k=1}^{r} T_{k} q\left(\lambda_{\max }(P 2) / \lambda_{\min }(P 1)\right), \ldots, c \beta_{1} \gamma_{J_{n}}+(1 / 2) \sum_{k=1}^{r} T_{k} q\left(\lambda_{\max }(P 2) / \lambda_{\min }(P 1)\right)\right.$, $\left.\left(3 c \gamma_{J_{d}}+\beta_{1}\right) \gamma_{J_{1}}, \ldots,\left(3 c \gamma_{J_{d}}+\beta_{1}\right) \gamma_{J_{n}}\right]^{T}$. Based on the conditions that $M_{\sigma}>0$ in each time interval and $D>0\left(D_{i}>0\right.$, for all $\left.i\right)$, for the sufficient small $T_{i j}$ and $c$, it is easy to verify that there always exist $M_{\sigma}$ and $D$ to guarantee $Q$ is positive definite. Therefore, we have that

$$
\dot{V} \leq-\lambda_{\min }(Q)\|x\|^{2}+b\|y\|=-\lambda_{\min }(Q)\|x\|^{2}+b\|x\|,
$$

where $b=\sqrt{\sum_{i=1}^{n}\left(c \beta_{1} \gamma_{J_{i}}+(1 / 2) \sum_{k=1}^{r} T_{k} q\left(\lambda_{\max }(P 2) / \lambda_{\min }(P 1)\right)\right)^{2}+\sum_{i=1}^{n} \gamma_{J_{i}}^{2}\left(3 c \gamma_{J_{d}}+\beta_{1}\right)^{2}}$. Thus, for $0<\theta<1$, if $\|x\| \geq b / \theta \lambda_{\min }(Q)$, we have that

$$
\dot{V}=-(1-\theta) \lambda_{\min }(Q)\|x\|^{2}-\theta \lambda_{\min }(Q)\|x\|^{2}+b\|x\| \leq-(1-\theta) \lambda_{\min }(Q)\|x\|^{2} .
$$

Therefore, the uniformly ultimate boundedness of $x$ follows from Lemma 3.4. In addition, the ultimate bound is $\lambda_{\max }(P 2) b / \lambda_{\min }(P 1) \theta \lambda_{\min }(Q)$ by following a similar analysis to that in [28]. 


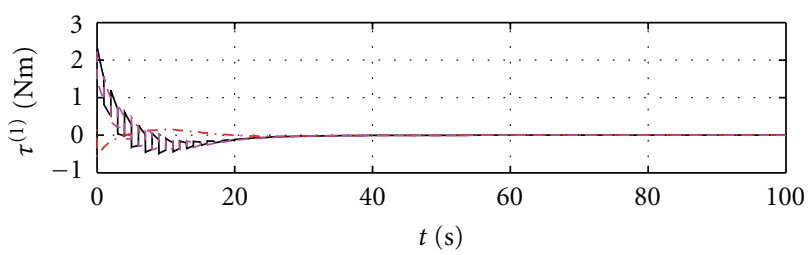

(a)

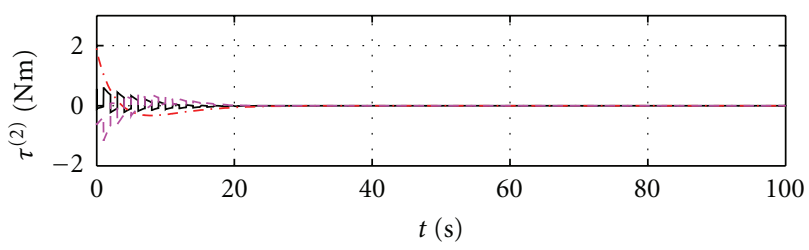

(b)

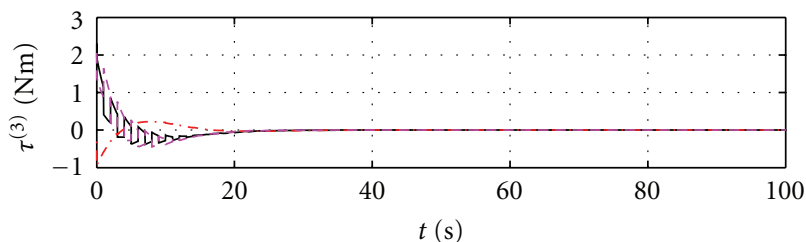

$-\mathrm{F} 1$
$-\ldots \mathrm{F} 2$
$-\ldots \mathrm{F} 3$

(c)

Figure 7: Rigid body control torques with control law (4.4).

Remark 6.3. Note that both the case of cooperative regulation and that of cooperative tracking discussed in this paper introduce model-independent control laws. The final errors converge to zero for the cooperative regulation case while the final errors are bounded for the cooperative tracking case. The authors in [26] showed that the final errors will be decreased effectively if the control parameters are chosen large enough for the tracking case. Similar conclusion also holds for our control law.

Remark 6.4. Note that the stability or uniformly ultimate boundedness conditions given in Theorems 5.2 and 6.2 are just sufficient conditions, not the necessary conditions.

Remark 6.5. Note that the bounds of the communication delays to guarantee the stability or uniformly ultimate boundedness of the closed-loop systems are implied in the conditions that $W>0$ for the regulation case and $Q>0$ for the tracking case. Also note that the bounds of the communication delays are related to the control parameters and their relationship is indirect.

Remark 6.6. Reference [17] also discussed the cooperative attitude tracking problem in the presence of communication delays and dynamically changing topologies, where stability result was obtained by using Lyapunov-Krasovskii Theorem. In contrast, here we use Lyapunov-Razumikhin Theorem to derive uniformly ultimate boundedness results of the closed-loop systems. 
Table 1: Spacecraft specifications.

\begin{tabular}{lc}
\hline$J_{1}$ & {$[23.00 .10 .1 ; 0.122 .20 .1 ; 0.10 .123 .2] \mathrm{kg} \cdot \mathrm{m}^{2}$} \\
$J_{2}$ & {$[22.50 .1-0.3 ; 0.122 .10 .1 ;-0.30 .124 .1] \mathrm{kg} \cdot \mathrm{m}^{2}$} \\
$J_{3}$ & {$[24.0-0.20 .1 ; 021.20 .1 ; 0.1-0.122 .2] \mathrm{kg} \cdot \mathrm{m}^{2}$} \\
\hline
\end{tabular}

\section{Simulation}

In this section, control laws (4.3) and (4.4) are used in simulation to achieve cooperative attitude regulation and tracking among three follower spacecraft. The spacecraft specifications are given in Table 1.

For control law (4.3), we choose the control parameters as $K_{i}=2, D_{i}=5$, and $l_{i j}=0.3$, for all $i, j \cdot q_{i}(0)$ and $\omega_{i}(0), i=1,2,3$ are generated randomly. For control law (4.4), we choose the control parameters as $K_{i}=2.2$ and $D_{i}=11 . q_{i}(0)$ and $\omega_{i}(0), i=1,2,3$ are generated randomly. Suppose that the reference attitude $q_{d}(t)$, reference angular velocity $\omega_{d}(t)=2 E^{-1}\left(q_{d}\right) \dot{q}_{d}$, reference torque $\tau_{d}$ and reference inertia satisfy (2.2) with $q_{d}(0)=$ $[0.3921,0.5502,0.5287,0.5139]^{T}$ (the corresponding Euler Angles are $\psi=80 \mathrm{deg}, \phi=60 \mathrm{deg}$, and $\theta=40 \mathrm{deg}), \omega_{d}(0)=[0.021,-0.012,0.014]^{T} \mathrm{rad} / \mathrm{s}, \tau_{d}=[0,0,0]^{T} \mathrm{Nm}$, and $J_{d}=$ $[22.0,0.2,-0.1 ; 0.2,23.1,0.3 ;-0.1,0.3,21.3] \mathrm{kg} \mathrm{m}^{2}$. For communication topology, we assume that $a_{i j}=1$ if $(j, i) \in \mathcal{E}$, and $a_{i j}=0$ otherwise. For control law (4.3), the communication topology for follower spacecraft is fixed and determined by $\mathcal{G}_{1}$ in Figure 1(a). For control law (4.4), the communication topology for follower spacecraft is switching between $\mathcal{G}_{1}$ in Figure 1(a) and $\mathcal{G}_{2}$ in Figure 1(b) every one second. The time-varying communication delays are chosen as $T_{12}=T_{21}=0.4|\sin (0.2 t)|, T_{13}=T_{31}=0.3|\cos (0.4 t)|$, and $T_{23}=T_{32}=$ $0.5|\sin (0.3 t)|$.

Figures 2, 3, and 4 show, respectively, the attitudes, angular velocities, and control torques of follower spacecraft 1,2, and 3 using (4.3) for (2.2). We can see from the figures that if the control parameters are selected properly, all spacecraft can regulate their attitude and angular velocity to zero even if there exists multiple time-varying communication delays.

Figures 5, 6 and 7 show, respectively, the attitudes, angular velocities and control torques of follower spacecraft 1, 2 and 3 using (4.4) for (2.2). We can see from the figures that if the control parameters are selected properly, all spacecraft can track time-varyingly desired attitude and angular velocity even if there exists multiple time-varying communication delays and dynamically changing topologies.

\section{Conclusions}

In this paper, the cooperative attitude regulation problem in the presence of multiple timevarying communication delays and the cooperative attitude tracking problem in the presence of multiple time-varying communication delays and dynamically changing topologies are discussed. Lyapunov-Krasovskii Theorem and Lyapunov-Razumikhin Theorem are used to derive the conditions to guarantee the stability or uniformly ultimate boundedness of the closed-loop system. Simulation results validate the effectiveness of the theoretical results. Future work will include proposing a more practical design by addressing the sign ambiguity problem for the unit quaternion description and discussing the cooperative attitude regulation problem in the presence of both communication delays and dynamically changing topologies. 


\section{References}

[1] W. Ren, R. W. Beard, and E. M. Atkins, "Information consensus in multivehicle cooperative control: collective group behavior through local interaction," IEEE Control Systems Magazine, vol. 27, no. 2, pp. 71-82, 2007.

[2] P. K. C. Wang and F. Y. Hadaegh, "Coordination and control of multiple microspacecraft moving in formation," Journal of the Astronautical Sciences, vol. 44, no. 3, pp. 315-355, 1996.

[3] R. S. Smith and F. Y. Hadaegh, "Distributed estimation, communication and control for deep space formations," IET Control Theory and Applications, vol. 1, no. 2, pp. 445-451, 2007.

[4] D. P. Scharf, F. Y. Hadaegh, and S. R. Ploen, "A survey of spacecraft formation flying guidance and control (part II): control," in Proceedings of the American Control Conference, vol. 4, pp. 2976-2985, Boston, Mass, USA, July 2004.

[5] P. K. C. Wang, F. Y. Hadaegh, and K. Lau, "Synchronized formation rotation and attitude control of multiple free-flying spacecraft," Journal of Guidance, Control, and Dynamics, vol. 22, no. 1, pp. 28-35, 1999.

[6] J. R. Lawton and R. W. Beard, "Synchronized multiple spacecraft rotations," Automatica, vol. 38, no. 8, pp. 1359-1364, 2002.

[7] W. Ren and R. W. Beard, "Decentralized scheme for spacecraft formation flying via the virtual structure approach," Journal of Guidance, Control, and Dynamics, vol. 27, no. 1, pp. 73-82, 2004.

[8] W. Ren, "Distributed attitude alignment in spacecraft formation flying," International Journal of Adaptive Control and Signal Processing, vol. 21, no. 2-3, pp. 95-113, 2007.

[9] M. C. Vandyke and C. D. Hall, "Decentralized coordinated attitude control within a formation of spacecraft," Journal of Guidance, Control, and Dynamics, vol. 29, no. 5, pp. 1101-1109, 2006.

[10] A. Abdessameud and A. Tayebi, "Attitude synchronization of a spacecraft formation without velocity measurement," in Proceedings of the 47th IEEE Conference on Decision and Control, pp. 3719-3724, Cancun, Mexico, December 2008.

[11] D. V. Dimarogonas, P. Tsiotras, and K. J. Kyriakopoulos, “Leader-follower cooperative attitude control of multiple rigid bodies," Systems \& Control Letters, vol. 58, no. 6, pp. 429-435, 2009.

[12] R. Olfati-Saber and R. M. Murray, "Consensus problems in networks of agents with switching topology and time-delays," IEEE Transactions on Automatic Control, vol. 49, no. 9, pp. 1520-1533, 2004.

[13] P. Lin, Y. Jia, J. Du, and F. Yu, "Average consensus for networks of continuous-time agents with delayed information and jointly-connected topologies," in Proceedings of the American Control Conference, pp. 3884-3889, St. Louis, Mo, USA, June 2009.

[14] Y. G. Sun and L. Wang, "Consensus of multi-agent systems in directed networks with uniform timevarying delays," IEEE Transactions on Automatic Control, vol. 54, no. 7, pp. 1607-1613, 2009.

[15] U. Münz, A. Papachristodoulou, and F. Allgöwer, "Delay-dependent rendezvous and flocking of large scale multi-agent systems with communication delays," in Proceedings of the 47th IEEE Conference on Decision and Control, pp. 2038-2043, Cancun, Mexico, December 2008.

[16] E. Jin, X. Jiang, and Z. Sun, "Robust decentralized attitude coordination control of spacecraft formation," Systems \& Control Letters, vol. 57, no. 7, pp. 567-577, 2008.

[17] J. Erdong and S. Zhaowei, "Robust attitude synchronisation controllers design for spacecraft formation," IET Control Theory \& Applications, vol. 3, no. 3, pp. 325-339, 2009.

[18] N. Chopra and M. W. Spong, Advances in Robot Control: Passivity-Based Control of Multi-Agent Systems, Springer, Berlin, Germany, 2007.

[19] P. F. Hokayem, D. M. Stipanović, and M. W. Spong, "Semiautonomous control of multiple networked Lagrangian systems," International Journal of Robust and Nonlinear Control, vol. 19, no. 18, pp. 2040 2055, 2009.

[20] S.-I. Niculescu, Delay Effects on Stability: A Robust Control Approach, Springer, London, UK, 2003.

[21] H. Schaub and J. L. Junkins, Analytical Mechanics of Space Systems, American Institute of Aeronautics and Astronautics, 2003

[22] F. R. K. Chung, Spectral Graph Theory, vol. 92, American Mathematical Society, 1997.

[23] J. K. Hale and S. M. Verduyn Lunel, Introduction to Functional-Differential Equations, vol. 99 of Applied Mathematical Sciences, Springer, New York, NY, USA, 1993.

[24] W. Kang and H. H. Yeh, "Coordinated attitude control of multisatellite systems," International Journal of Robust Nonlinear Control, vol. 12, no. 2-3, pp. 185-205, 2002.

[25] J. Hu and Y. Hong, "Leader-following coordination of multi-agent systems with coupling time delays," Physica A, vol. 374, no. 2, pp. 853-863, 2007.

[26] J.-Y. Wen and K. Kreutz-Delgado, "The attitude control problem," IEEE Transactions on Automatic 
Control, vol. 36, no. 10, pp. 1148-1162, 1991.

[27] K. Peng and Y. Yang, "Leader-following consensus problem with a varying-velocity leader and timevarying delays," Physica A, vol. 388, no. 2-3, pp. 193-208, 2009.

[28] H. K. Khalil, Nonlinear Systems, Prentice Hall, Upper Saddle River, NJ, USA, 3rd edition, 2002. 


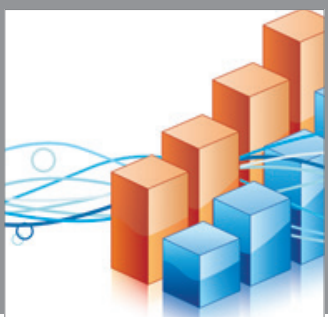

Advances in

Operations Research

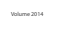

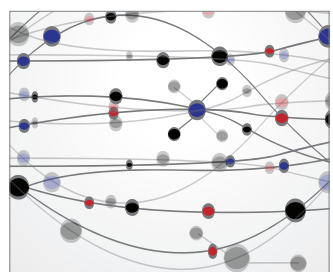

\section{The Scientific} World Journal
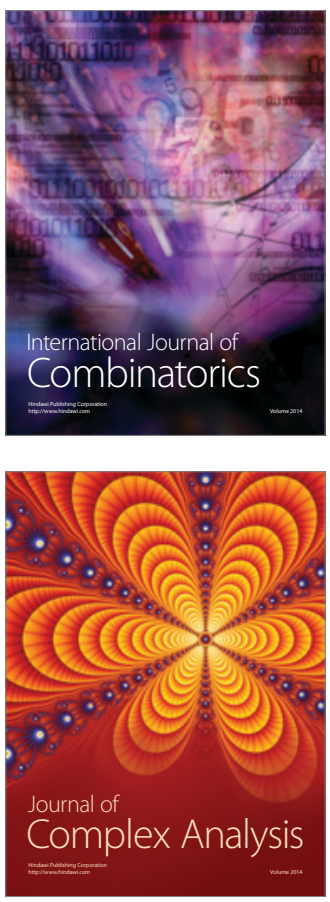

International Journal of

Mathematics and

Mathematical

Sciences
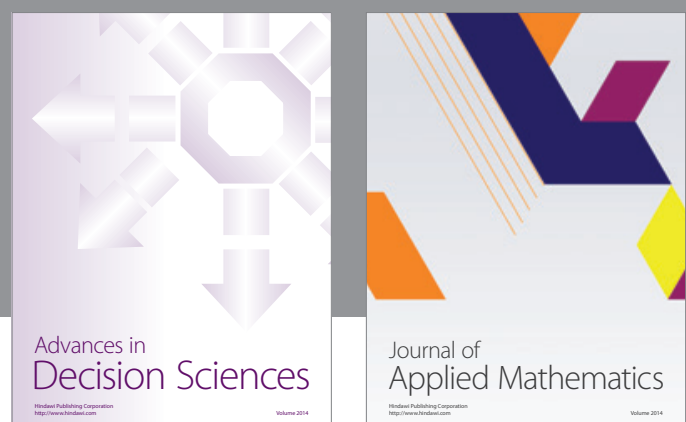

Journal of

Applied Mathematics
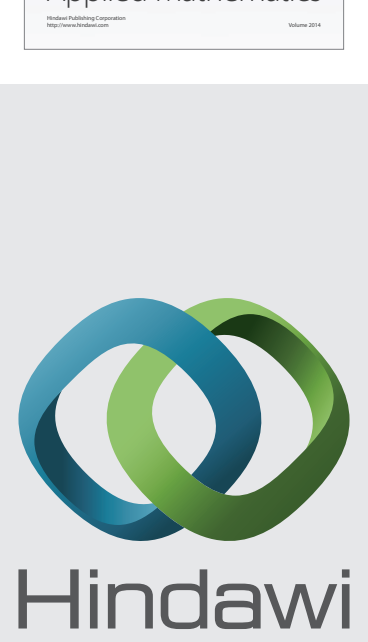

Submit your manuscripts at http://www.hindawi.com
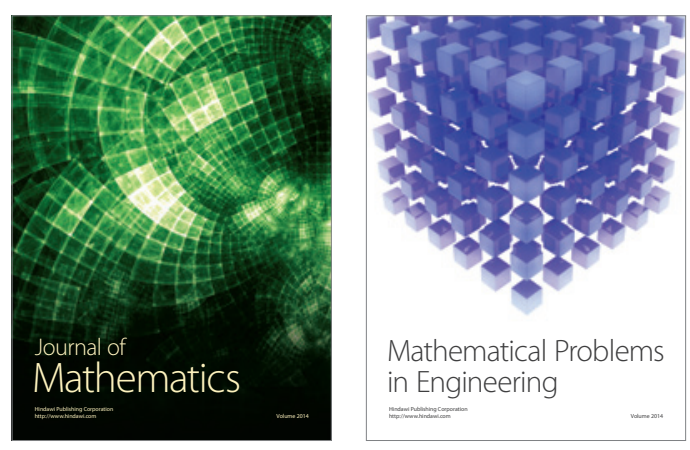

Mathematical Problems in Engineering
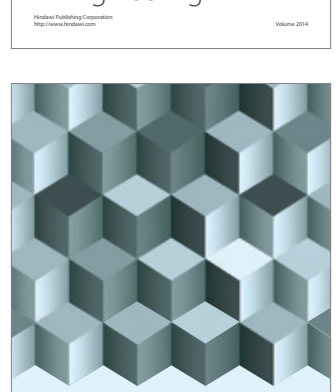

Journal of

Function Spaces
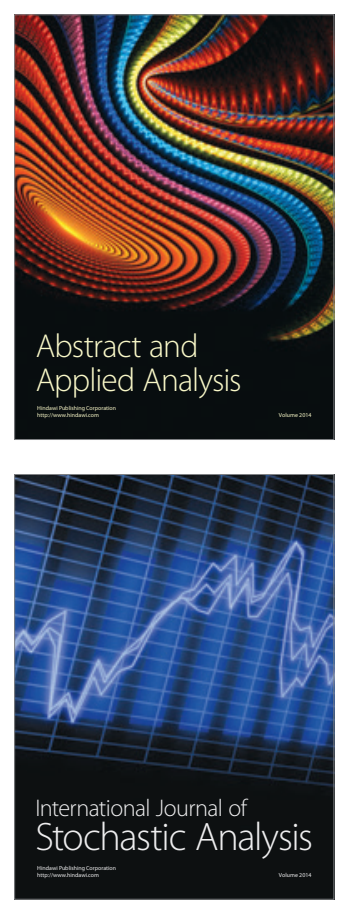

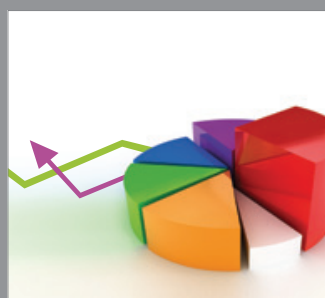

ournal of

Probability and Statistics

Promensencen
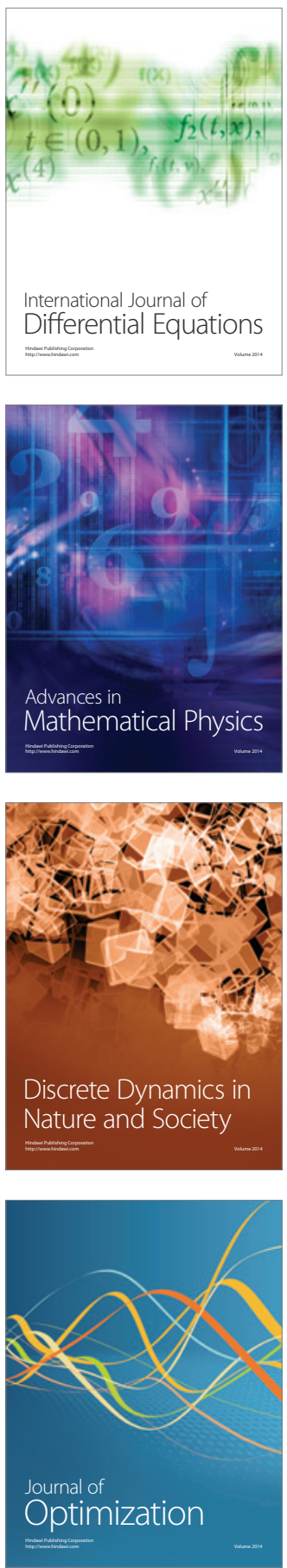